\title{
Review of: "From genes to public health: are we ready for DNA-based population screening?"
}

\author{
Arun Meyyazhagan ${ }^{1}$
}

1 University of Perugia

Potential competing interests: The author(s) declared that no potential competing interests exist.

1. As the reference template (The Human Genome Project) is still in progress, is it possible for health care providers to use DNA based screening for all patients to provide personalized therapy?

2. Increasing in the variants of unknown significance in the current medical era might hinder in identifying the cause of pathogenicity and can deviate the mode of therapy?

3. Based on the commentary evidence and recommendation based guidelines are limited to genetic conditions (HBOC, Lynch syndrome, and familial hypercholesterolemia). Before proceeding with the screening standardized guidelines are mandatory for other disorders and health conditions too. For example- diabetes mellitus, obesity, which are widely prevalent.

4. Few common pathologies might have the same symptoms like genetic conditions, so directly considering them for DNA screening wouldn't be a burden on the economy and the mental health of the individual.

5. Will the screening results can be interpretable easily by everyone? 